\title{
Cardinalities of Primitive Fixing Systems for Convex Bodies
}

\author{
V. Boltyanski and E. Morales Amaya \\ El Centro de Investigacion en Matematicas, \\ A.P. 402, 36000, Guanajuato, Mexico \\ boltian@fractal.cimat.mx
}

\begin{abstract}
We establish the exact upper bound of cardinalities of primitive fixing systems for any compact, convex body $M$, depending on $\operatorname{dim} M$ and $m d M$. In a sense, this is the last touch to the theory of fixing systems for compact, convex bodies.
\end{abstract}

\section{Introduction}

Fejes Tóth [10] formulated a problem on primitive fixing systems for convex bodies. Namely, let $M \subset R^{n}$ be a compact, convex body. A set $F \subset$ bd $M$ is a fixing system for $M$ [10] if for any nonzero vector $v$ there is a real number $\lambda>0$ such that the translate $\lambda v+$ int $M$ of the interior of $M$ has a point in common with $F$. A fixing system $F \subset$ bd $M$ is primitive if no proper subset $F^{\prime} \subset F$ is a fixing system for $M$. The minimal cardinality of fixing systems for $M$ is denoted here by $\varrho_{\min }(M)$, and the maximal cardinality of primitive fixing systems for $M$ is denoted by $\varrho_{\max }(M)$ (if there are primitive fixing systems for $M$ with any large integer cardinality, we write $\left.\varrho_{\max }(M)=\infty\right)$. We remark that every fixing system of minimal cardinality is primitive.

Fejes Tóth proved [10] that for two-dimensional compact, convex figures the maximal cardinality of primitive fixing systems is equal to 6 :

$$
3 \leq \varrho_{\min }(M) \leq \varrho_{\max }(M) \leq 6,
$$

the upper bound being attained for any hexagon with pairwise parallel opposite sides.

Danzer [9] conjectured that for three-dimensional compact, convex bodies the maximal cardinality of primitive fixing systems is equal to 14 (being attained for affine images of the rhombic dodecahedron). A counterexample was given by Bollobás [1], who constructed three-dimensional convex revolution bodies with primitive fixing systems of any large integer cardinalities. Simpler examples are given in [5] and [12].

For the cardinalities of minimal fixing systems the exact lower and upper bounds were found by Grünbaum [11]. Namely, for any compact, convex body $M \subset R^{n}$ the following 
inequalities hold:

$$
n+1 \leq \varrho_{\min }(M) \leq 2 n
$$

These estimates are exact, i.e., there exists a body (for example, a ball) for which the lower estimate is attained, and there exists a body (namely, a parallelotope) for which the upper bound is attained.

In [4] and [8] some more detailed estimates are given. To formulate these results, we recall the definition of the maximin dependence functional md $M$, considered on the family of all compact, convex bodies in $R^{n}$ and introduced in [2]. Namely, $m+1$ vectors $a_{0}, a_{1}, \ldots, a_{m} \in R^{n}$ are said to be minimally dependent if they are positively dependent (i.e., $\lambda_{0} a_{0}+\lambda_{1} a_{1}+\cdots+\lambda_{m} a_{m}=0$ with some positive $\lambda_{0}, \lambda_{1}, \ldots, \lambda_{m}$ ) and also every $m$ of them are linearly independent. Furthermore, for a compact, convex body $M \subset R^{n}$ denote by $H(M)$ the set of all unit outward normals of $M$ at its regular boundary points (a boundary point is regular if there is only one support hyperplane for $M$ through the point). By md $M$ [2] we denote the maximal integer $m$ such that there are minimally dependent vectors $a_{0}, a_{1}, \ldots, a_{m} \in H(M)$. It is proved in [2] and [3] that md $M$ is equal to the Helly dimension (denoted by him $M$ ) of the body $M$, i.e., $\operatorname{md} M$ is the least of the integers $m$ with the following property: if $M_{1}, \ldots, M_{q}$ are arbitrary translates of $M$ and every $m+1$ of the translates has a point in common, then $M_{1} \cap \cdots \cap M_{q} \neq \emptyset$.

The equality $\operatorname{him} M=1$ holds if and only if $M$ is a parallelotope [2], [13]. Furthermore, in [3] a complete list of compact, convex bodies with him $M=2$ is given. Namely, $\operatorname{him} M=2$ if and only if $M$ is a stack, an outcut, a stack-outcut, or a four-dimensional particular polytope. The definition of the stack (through its polar body) is given in the beginning of the proof of Lemma 2 and the definition of the four-dimensional particular polytope (again through its polar body) is given in the beginning of the proof of Lemma 3. As to the outcuts and stack-outcuts, they are defined analogously to the stacks (we refer the reader to [3] for detailed definitions).

Now we can formulate the results established in [4] and [8]: For any compact, convex body $M \subset R^{n}$ the estimates

$$
n+\frac{n}{\operatorname{md} M} \leq \varrho_{\min }(M) \leq 2 n+1-\operatorname{md} M
$$

hold, both the estimates being exact.

For maximal cardinalities of primitive fixing systems of any compact, convex body $M \subset R^{n}$ we only have the estimate

$$
\varrho_{\max }(M) \leq \infty
$$

that also is exact. This follows from [5] where an $n$-dimensional, compact, convex body is constructed with $\varrho_{\max }(M)=\infty$ (see also [12]). However, the estimate (4) is not written depending on md $M$. The problem of finding the estimates for maximal cardidalities of primitive fixing systems depending on $\operatorname{dim} M$ and $m d M$ was open, and this was the last problem from the considered circle of questions. This problem is solved in the next section. 


\section{Main Theorem}

The following theorem contains the exact upper bound of the cardinalities of primitive fixing systems for any compact, convex body $M$ depending on $\operatorname{dim} M$ and $\operatorname{md} M$.

Theorem. Let $M \subset R^{n}$ be a compact, convex body. If $\mathrm{md} M=1$ (i.e., $M$ is an $n$-dimensional parallelotope), then $\varrho_{\max }(M)=2 n$. If $\mathrm{md} M=2$, then

$$
\varrho_{\max }(M) \leq \frac{9}{2} n+\alpha_{n},
$$

where

$$
\begin{array}{llll}
\alpha_{n}=0 & \text { for } n \equiv 0 \quad(\bmod 4), & \alpha_{n}=-\frac{5}{2} & \text { for } n \equiv 1 \quad(\bmod 4), \\
\alpha_{n}=-3 & \text { for } n \equiv 2 \quad(\bmod 4), & \alpha_{n}=-\frac{11}{2} \quad \text { for } n \equiv 3 \quad(\bmod 4) .
\end{array}
$$

If md $M \geq 3$, then $\varrho_{\max }(M) \leq \infty$.

All the estimates are exact (i.e., there exists a compact, convex body $M \subset R^{n}$ with the prescribed $\mathrm{md} M$ for which the indicated upper bound is attained).

Thus, together with (3), we obtain the following exact estimates:

$$
\begin{gathered}
\varrho_{\text {min }}(M)=\varrho_{\text {max }}(M)=2 n \quad \text { for } \quad \operatorname{md} M=1, \\
\frac{3}{2} n \leq \varrho_{\text {min }}(M) \leq \varrho_{\text {max }}(M) \leq \frac{9}{2} n+\alpha_{n} \quad \text { for } \quad \operatorname{md} M=2, \\
n+\frac{n}{\operatorname{md} M} \leq \varrho_{\text {min }}(M) \leq \varrho_{\text {max }}(M) \leq \infty \quad \text { for } \quad \operatorname{md} M \geq 3 .
\end{gathered}
$$

We remark that for the case md $M=2$ not every integer contained between the indicated lower and upper bounds (see (8)) is the cardinality of a primitive fixing system for $M$ (see the remark at the end of the paper).

\section{Proof of the Main Theorem}

First, we describe the way to find the number $\varrho_{\max }(M)$ with the help of the polar body $M^{*}$. Let $M \subset R^{n}$ be a compact, convex body with the origin in its interior and let $M^{*}$ be its polar body, i.e.,

$$
M^{*}=\left\{y \in R^{n}:\langle y, x\rangle \leq 1 \text { for all } x \in M\right\} .
$$

Let $\Gamma_{1}, \ldots, \Gamma_{k}$ be support hyperplanes of $M^{*}$ and let $B_{i}=M^{*} \cap \Gamma_{i}, i=i, \ldots, k$. We say that $\left\{\Gamma_{1}, \ldots, \Gamma_{k}\right\}$ is a fixing cosystem for $M^{*}$ if for every open half-space $P \subset R^{n}$ with the boundary hyperplane bd $P$ through the origin, at least one of the sets $B_{1}, \ldots, B_{k}$ is completely contained in $P$. The fixing cosystem $\left\{\Gamma_{1}, \ldots, \Gamma_{k}\right\}$ is primitive if no proper subset of $\left\{\Gamma_{1}, \ldots, \Gamma_{k}\right\}$ is a fixing cosystem for $M^{*}$. By $\varrho_{\max }^{*}\left(M^{*}\right)$ we denote the maximal $k$ for which there exists a primitive fixing cosystem $\left\{\Gamma_{1}, \ldots, \Gamma_{k}\right\}$ for $M^{*}$ (if there are primitive fixing cosystems for $M^{*}$ with any large integer cardinality, we write $\varrho_{\max }^{*}\left(M^{*}\right)=\infty$ ). 
Lemma 1. For any compact, convex body $M \subset R^{n}$ containing the origin in its interior the equality $\varrho_{\max }^{*}\left(M^{*}\right)=\varrho_{\max }(M)$ holds.

Proof. Let $F=\left\{x_{1}, \ldots, x_{k}\right\} \subset$ bd $M$ be a fixing system for the body $M$. Denote by $\Gamma_{1}, \ldots, \Gamma_{k}$ the polar hyperplanes for the points $x_{1}, \ldots, x_{k}$, i.e.,

$$
\Gamma_{i}=x_{i}^{*}=\left\{y \in R^{n}:\left\langle y, x_{i}\right\rangle=1\right\}, \quad i=1, \ldots, k .
$$

Then $\Gamma_{1}, \ldots, \Gamma_{k}$ are support hyperplanes of the polar body $M^{*}$. It is easily shown that $\left\{\Gamma_{1}, \ldots, \Gamma_{k}\right\}$ is a fixing cosystem for $M^{*}$. Indeed, let $P \subset R^{n}$ be an open half-space with the boundary hyperplane through the origin. Denote by $l$ the direction defined by the outward normal $p$ of the half-space $P$. Since $F$ is a fixing system for the body $M$, there exists a point $x_{i} \in F$ that is illuminated by the direction $l$ (see Theorem 17 in [4]), i.e., there is $\lambda>0$ such that the point $x_{\lambda}=x_{i}+\lambda p$ belongs to the interior of $M$. We may suppose (diminishing $\lambda$ if necessary) that $x_{\lambda}$ is distinct from the origin. Let $z$ be a point of the set $B_{i}=M^{*} \cap \Gamma_{i}$. Since $z \in \Gamma_{i}$, we have

$$
\left\langle z, x_{i}\right\rangle=1
$$

Furthermore, since $x_{\lambda}$ is an interior point of the body $M$, the polar hyperplane $x_{\lambda}^{*}$ has no points in common with $M^{*}$, i.e., the body $M^{*}$ is situated in the half-space $\left\{y:\left\langle y, x_{\lambda}\right\rangle<1\right\}$. In particular,

$$
\left\langle z, x_{\lambda}\right\rangle<1
$$

From (9) and (10) we obtain $\left\langle z, x_{\lambda}-x_{i}\right\rangle<0$, i.e., $\langle z, \lambda p\rangle<0$, and hence $\langle z, p\rangle<0$ (since $\lambda>0$ ). This means that $z \in P$. The obtained inclusion holds for any point $z \in B_{i}$, i.e., $B_{i} \subset P$. Thus $\left\{\Gamma_{1}, \ldots, \Gamma_{k}\right\}$ is a fixing cosystem for $M^{*}$.

Conducting the above reasoning in the opposite direction, we find that, conversely, if $\left\{\Gamma_{1}, \ldots, \Gamma_{k}\right\}$ is a fixing cosystem for $M^{*}$, then the corresponding polar points $x_{1}, \ldots, x_{k}$ (i.e., the points with $x_{i}^{*}=\Gamma_{i}, i=1, \ldots, k$ ) form a fixing system for the body $M$. This implies that the fixing cosystem $\left\{\Gamma_{1}, \ldots, \Gamma_{k}\right\}$ is primitive if and only if the fixing system $\left\{x_{1}, \ldots, x_{k}\right\}$ is primitive.

In the following two lemmas we conserve the terminology introduced in Theorems 29.1 and 29.7 of [7].

Lemma 2. Let $M \subset R^{n}$ be an n-dimensional stack, outcat, or stack-outcat. Then $\varrho_{\max }(M) \leq 2 n$.

Proof. We investigate in detail the case when $M$ is an $n$-dimensional stack (the cases of outcat and stack-outcat being similar).

A compact, convex body $M \subset R^{n}$ is said to be an $n$-dimensional stack if the corresponding polar body $M^{*}$ is described in the following way (see case (B) of Theorem 29.1 in [7]). There exists a basis $e_{1}, \ldots, e_{n}$ in $R^{n}$ and closed, two-dimensional, convex sets 
$C_{2}, \ldots, C_{n}$ such that

(i) $e_{1} \in C_{l} \subset \operatorname{lin}\left(e_{1}, e_{l}\right)$ and ext $C_{l} \subset\left\{e_{1}\right\} \cup \Pi_{l, 1}$ for $l=2, \ldots, n$, where $\Pi_{l, 1}$ is the half-plane $\left\{v: v=x_{1} e_{1}+x_{l} e_{l}, x_{1} \leq 0\right\}$

(ii) $M^{*}=\operatorname{conv}\left(C_{2} \cup \cdots \cup C_{n}\right)$.

For each $l=2, \ldots, n$ denote by $F_{l}^{+}$the angle $\left\{v: v=x_{1} e_{1}+x_{l} e_{l}, x_{1} \leq 0, x_{l}>0\right\}$, and by $F_{l}^{-}$the angle $\left\{v: v=x_{1} e_{1}+x_{l} e_{l}, x_{1} \leq 0, x_{l}<0\right\}$.

Let $\left\{\Gamma_{1}, \ldots, \Gamma_{k}\right\}$ be a fixing cosystem for $M^{*}$ and $B_{1}=M^{*} \cap \Gamma_{1}, \ldots, B_{k}=M^{*} \cap \Gamma_{k}$. For arbitrary $l=2, \ldots, n$ denote by $P_{l}^{+} \subset R^{n}$ the open half-space consisting of all vectors $x_{1} e_{1}+\cdots+x_{n} e_{n}$ with $x_{l}>0$. Then at least one of the sets $B_{1}, \ldots, B_{k}$ is contained in $P_{l}^{+}$. Denote such a set by $B_{l}^{+}$. Similarly, for arbitrary $l=2, \ldots, n$ denote by $P_{l}^{-} \subset R^{n}$ the open half-space consisting of all vectors $x_{1} e_{1}+\cdots+x_{n} e_{n}$ with $x_{l}<0$. Then at least one of the sets $B_{1}, \ldots, B_{k}$ is contained in $P_{l}^{-}$. Denote such a set by $B_{l}^{-}$. We remark that $B_{l}^{+}$(respectively, $B_{l}^{-}$) is a point or a segment contained in bd $M^{*} \cap F_{l}^{+}$ (respectively, in bd $M^{*} \cap F_{l}^{-}$).

Finally, denote by $P_{1}^{+}$(respectively, $P_{1}^{-}$) the open half-space consisting of all vectors $x_{1} e_{1}+\cdots+x_{n} e_{n}$ with $x_{1}>0$ (respectively, $x_{1}<0$ ). Then at least one of the sets $B_{1}, \ldots, B_{k}$ is contained in $P_{1}^{+}$, and it is easily shown that this set $B_{1}^{+}$coincides with $\left\{e_{1}\right\}$.

Thus we already have $2 n-1$ different sets $B_{1}^{+}, B_{l}^{+}, B_{l}^{-}, l=2, \ldots, n$, taken from $B_{1}, \ldots, B_{k}$ and hence $k \geq 2 n-1$. Furthermore, let $P \subset R^{n}$ be an open half-space with the boundary hyperplane through the origin. If $e_{1} \notin P$ and $P \neq P_{1}^{-}$, then $P$ contains at least one of the angles $F_{2}^{+}, \ldots, F_{n}^{+}, F_{2}^{-}, \ldots, F_{n}^{-}$. Consequently it contains at least one of the sets $B_{l}^{+}, B_{l}^{-}, l=2, \ldots, n$. If even $e_{1} \in P$, then $P$ contains the set $B_{1}^{+}=\left\{e_{1}\right\}$. Thus every open half-space $P$ with $0 \in$ bd $P$ distinct from $P_{1}^{-}$contains at least one of the sets $B_{1}^{+}, B_{l}^{+}, B_{l}^{-}, l=2, \ldots, n$.

If now the half-space $P_{1}^{-}$contains at least one of the sets $B_{l}^{+}, B_{l}^{-}, l=2, \ldots, n$, then any open half-space of $R^{n}$ with the boundary hyperplane through the origin contains at least one of the sets $B_{1}^{+}, B_{l}^{+}, B_{l}^{-}, l=2, \ldots, n$, i.e., we have a fixing cosystem for $M^{*}$ taken from $\left\{\Gamma_{1}, \ldots, \Gamma_{k}\right\}$ that contains $2 n-1$ hyperplanes. In this case $k=2 n-1$. Even if the half-space $P_{1}^{-}$does not contain any one of the sets $B_{l}^{+}, B_{l}^{-}, l=2, \ldots, n$, then it is necessary to add one more of the sets $B_{1}, \ldots, B_{k}$, i.e., $k=2 n$. Thus in any case $\varrho_{\max }^{*}\left(M^{*}\right) \leq 2 n$.

By Lemma 1, we conclude that $\varrho_{\max }(M) \leq 2 n$.

Remark. In the condition of Lemma 2 , the equality $\varrho_{\max }(M)=2 n$ is possible. For example, let $M_{1}$ be an $n$-dimensional cube and let $M_{2}$ be the $n$-dimensional right pyramid whose basis coincides with a facet $F$ of the cube $M_{1}$ and the polytopes $M_{1}, M_{2}$ have no interior point in common. Then $\varrho_{\max }\left(M_{1} \cup M_{2}\right)=2 n$. Indeed, taking a point in the relative interior of every facet of the cube $M_{1}$ except $F$ (having thus $2 n-1$ points) and adding the vertex of the pyramid $M_{2}$ that is opposite to its basis, we obtain a primitive fixing system for the stack $M_{1} \cup M_{2}$ that consists of $2 n$ points.

Lemma 3. Let $M \subset R^{4}$ be the particular four-dimensional polytope with $\mathrm{md} M=2$. Then $\varrho_{\max }(M)=18$. 
Proof. A polytope $M \in R^{4}$ is said to be a particular one if the corresponding polar polytope $M^{*}$ is described in the following way (see case (D) of Theorem 29.1 in [7]). There is a basis $e_{1}, e_{2}, e_{3}, e_{4}$ of the space $R^{4}$ such that the polar polytope $M^{*}$ is the convex hull of the set consisting of the following nine points:

$$
\begin{gathered}
e_{1}, e_{2}, e_{3}, e_{4}, e_{1}+e_{2}+e_{3}+e_{4}, \\
-e_{1}-e_{2},-e_{1}-e_{3},-e_{2}-e_{4},-e_{3}-e_{4} .
\end{gathered}
$$

In the coordinate system with the basis $e_{1}, e_{2}, e_{3}, e_{4}$, consider the following nine hyperplanes in $R^{4}$ :

$$
\begin{gathered}
\Delta_{1}=\left\{x: x_{1}=0\right\}, \quad \Delta_{2}=\left\{x: x_{2}=0\right\}, \\
\Delta_{3}=\left\{x: x_{3}=0\right\}, \quad \Delta_{4}=\left\{x: x_{4}=0\right\}, \\
\Delta_{5}=\left\{x: x_{1}-x_{2}=0\right\}, \quad \Delta_{6}=\left\{x: x_{1}-x_{3}=0\right\}, \quad \Delta_{7}=\left\{x: x_{2}-x_{4}=0\right\}, \\
\Delta_{8}=\left\{x: x_{3}-x_{4}=0\right\}, \quad \Delta_{9}=\left\{x: x_{1}-x_{2}-x_{3}+x_{4}=0\right\} .
\end{gathered}
$$

Each of these hyperplanes defines two half-spaces. Thus we obtain the following 18 open half-spaces:

$$
\begin{gathered}
P_{1}^{+}=\left\{x: x_{1}>0\right\}, \quad P_{2}^{+}=\left\{x: x_{2}>0\right\}, \\
P_{3}^{+}=\left\{x: x_{3}>0\right\}, \quad P_{4}^{+}=\left\{x: x_{4}>0\right\}, \\
P_{5}^{+}=\left\{x: x_{1}-x_{2}>0\right\}, \quad P_{6}^{+}=\left\{x: x_{1}-x_{3}>0\right\}, \quad P_{7}^{+}=\left\{x: x_{2}-x_{4}>0\right\}, \\
P_{8}^{+}=\left\{x: x_{3}-x_{4}>0\right\}, \quad P_{9}^{+}=\left\{x: x_{1}-x_{2}-x_{3}+x_{4}>0\right\}, \\
P_{1}^{-}=\left\{x: x_{1}<0\right\}, \quad P_{2}^{-}=\left\{x: x_{2}<0\right\}, \\
P_{3}^{-}=\left\{x: x_{3}<0\right\}, \quad P_{4}^{-}=\left\{x: x_{4}<0\right\}, \\
P_{5}^{-}=\left\{x: x_{1}-x_{2}<0\right\}, \quad P_{6}^{-}=\left\{x: x_{1}-x_{3}<0\right\}, \quad P_{7}^{-}=\left\{x: x_{2}-x_{4}<0\right\}, \\
P_{8}^{-}=\left\{x: x_{3}-x_{4}<0\right\}, \quad P_{9}^{-}=\left\{x: x_{1}-x_{2}-x_{3}+x_{4}<0\right\} .
\end{gathered}
$$

Every hyperplane $\Delta_{j}, j=1, \ldots, 9$, contains five vertices of the polytope $M^{*}$. For example, the hyperplane $\Delta_{1}$ contains the vertices $e_{2}, e_{3}, e_{4},-e_{2}-e_{4},-e_{3}-e_{4}$. Furthermore, each of the half-spaces $P_{j}^{+}$and $P_{j}^{-}, j=1, \ldots, 9$, contains exactly two vertices of $M^{*}$. For example, the half-space $P_{1}^{+}$contains the vertices $e_{1}$ and $e_{1}+e_{2}+e_{3}+e_{4}$ which are situated in the support hyperplane $\left\{x: x_{1}=1\right\}$ of the polytope $M^{*}$. Consequently, $P_{1}^{+}$ contains the edge $I_{1}^{+}$of $M^{*}$ with the endpoints $e_{1}$ and $e_{1}+e_{2}+e_{3}+e_{4}$.

Similarly, every half-space $P_{j}^{+}$(respectively, $P_{j}^{-}$), $j=1, \ldots, 9$, contains one edge $I_{j}^{+}$(respectively, $I_{j}^{-}$) of the polytope $M^{*}$. Thus we obtain the following 18 edges of the polytope $M^{*}$ :

$$
\begin{aligned}
I_{1}^{+} & =\left[e_{1}, e_{1}+e_{2}+e_{3}+e_{4}\right], & & I_{1}^{-}=\left[-e_{1}-e_{2},-e_{1}-e_{3}\right], \\
I_{2}^{+} & =\left[e_{2}, e_{1}+e_{2}+e_{3}+e_{4}\right], & I_{2}^{-} & =\left[-e_{1}-e_{2},-e_{2}-e_{4}\right], \\
I_{3}^{+} & =\left[e_{3}, e_{1}+e_{2}+e_{3}+e_{4}\right], & & I_{3}^{-}=\left[-e_{1}-e_{3},-e_{3}-e_{4}\right], \\
I_{4}^{+} & =\left[e_{4}, e_{1}+e_{2}+e_{3}+e_{4}\right], & & I_{4}^{-}=\left[-e_{2}-e_{4},-e_{3}-e_{4}\right], \\
I_{5}^{+} & =\left[e_{1},-e_{2}-e_{4}\right], & & I_{5}^{-}=\left[e_{2},-e_{1}-e_{3}\right], \\
I_{6}^{+} & =\left[e_{1},-e_{3}-e_{4}\right], & & I_{6}^{-}=\left[e_{3},-e_{1}-e_{2}\right], \\
I_{7}^{+} & =\left[e_{2},-e_{3}-e_{4}\right], & & I_{7}^{-}=\left[e_{4},-e_{1}-e_{2}\right], \\
I_{8}^{+} & =\left[e_{3},-e_{2}-e_{4}\right], & & I_{8}^{-}=\left[e_{4},-e_{1}-e_{3}\right], \\
I_{9}^{+} & =\left[e_{1}, e_{4}\right], & I_{9}^{-} & =\left[e_{2}, e_{3}\right] .
\end{aligned}
$$


It is easily shown that every open half-space $P$ with the boundary hyperplane through the origin contains at least one of the indicated 18 segments. Indeed, let $P=\left\{x: a_{1} x_{1}+\right.$ $\left.a_{2} x_{2}+a_{3} x_{3}+a_{4} x_{4}>0\right\}$ be an open half-space in $R^{4}$ (at least one of the numbers $a_{1}, a_{2}, a_{3}, a_{4}$ being distinct from 0 ). If $a_{1}+a_{2}+a_{3}+a_{4}>0$, then at least one of the coefficients $a_{1}, a_{2}, a_{3}, a_{4}$ is positive. This implies that at least one of the segments $I_{1}^{+}, I_{2}^{+}, I_{3}^{+}, I_{4}^{+}$is contained in $P$. Let now $a_{1}+a_{2}+a_{3}+a_{4} \leq 0$. If $a_{1}$ is the only positive coefficient, then at least one of the segments $I_{5}^{+}, I_{6}^{+}$is contained in $P$. In general, if only one of the coefficients $a_{1}, a_{2}, a_{3}, a_{4}$ is positive, then at least one of the segments $I_{j}^{+}, I_{j}^{-}, j=5,6,7,8$, is situated in $P$. Furthermore, if $a_{1}$ and $a_{2}$ are the only positive coefficients, then $a_{3}+a_{4}<0$ and hence $I_{6}^{+} \subset P$. More general, if there are two or three positive coefficients (under the condition $a_{1}+a_{2}+a_{3}+a_{4} \leq 0$ ), then at least one of the segments $I_{j}^{+}, I_{j}^{-}, j=5, \ldots 9$, is contained in $P$. If now all the coefficients are nonpositive, then at least one of the segments $I_{j}^{-}, j=1,2,3,4$, is contained in $P$. Thus in any case at least one of the segments $I_{j}^{+}, I_{j}^{-}, j=1, \ldots, 9$, is situated in $P$.

Let $\Sigma_{j}^{+}$(respectively, $\Sigma_{j}^{-}$) be a support hyperplane of the body $M^{*}$ with $\Sigma_{j}^{+} \cap M^{*}=$ $I_{j}^{+}$(respectively, $\Sigma_{j}^{-} \cap M^{*}=I_{j}^{-}$), $j=1, \ldots, 9$. Then the hyperplanes $\Sigma_{j}^{+}, \Sigma_{j}^{-}$, $j=1, \ldots, 9$, form a fixing cosystem for $M^{*}$ (since every open half-space $P$ with the boundary hyperplane through the origin contains at least one of the indicated $18 \mathrm{seg}$ ments). Moreover, this fixing cosystem is primitive (since for each one of the 18 segments there is a half-space that contains only the chosen segment). Thus $\varrho_{\max }^{*}\left(M^{*}\right) \geq 18$.

Now we establish the opposite inequality. Let $\Gamma_{1}, \ldots, \Gamma_{k}$ be a fixing cosystem for $M^{*}$ and let $B_{i}=M^{*} \cap \Gamma_{i}, i=1, \ldots, k$. Then every open half-space $P$ with the boundary hyperplane through the origin contains at least one of the sets $B_{1}, \ldots, B_{k}$. Hence at least one of the sets $B_{1}, \ldots, B_{k}$ is contained in the half-space $P_{j}^{+}$and at least one in $P_{j}^{-}, j=1, \ldots, 9$. Denote such a set by $B_{j}^{+}$(respectively, $B_{j}^{-}$). Since the segment $I_{j}^{+}$(respectively $I_{j}^{-}$) is the unique face of the polytope $M^{*}$ in the half-space $P_{j}^{+}$(respectively, $P_{j}^{-}$), we have $B_{j}^{+} \subset I_{j}^{+}$(respectively, $\left.B_{j}^{-} \subset I_{j}^{-}\right), j=1, \ldots, 9$. The corresponding hyperplane (taken from $\Gamma_{1}, \ldots, \Gamma_{k}$ ) denote by $\Gamma_{j}^{+}$(respectively, $\Gamma_{j}^{-}$), $j=1, \ldots, 9$.

Thus we have 18 sets $B_{j}^{+}, B_{j}^{-}, j=1, \ldots, 9$, taken from $B_{1}, \ldots, B_{k}$ (it is possible that some of them are coincident, and hence we have less than 18 hyperplanes $\Gamma_{j}^{+}, \Gamma_{j}^{-}$). The hyperplanes $\Gamma_{j}^{+}, \Gamma_{j}^{-}, j=1, \ldots, 9$, form a fixing cosystem for the body $M^{*}$ (since every open half-space $P$ with the boundary hyperplane through the origin contains at least one of the above 18 segments and hence contains at least one of the sets $B_{j}^{+}, B_{j}^{-}$, $j=1, \ldots, 9)$. Thus no more than 18 hyperplanes taken from $\Gamma_{1}, \ldots, \Gamma_{k}$ form a fixing cosystem for $M^{*}$, and hence $k \leq 18$ (by primitivity of the cosystem $\left\{\Gamma_{1}, \ldots, \Gamma_{k}\right\}$ ), i.e., $\varrho_{\max }^{*}\left(M^{*}\right) \leq 18$. Consequently, $\varrho_{\max }^{*}\left(M^{*}\right)=18$.

By Lemma 1, we conclude that $\varrho_{\max }(M)=18$.

Now we are able to prove the main theorem. For $m d M=1$ (i.e., for the case when $M$ is an $n$-dimensional parallelotope) any primitive fixing system consists of $2 n$ points situated in relative interiors of the facets of the parallelotope (each facet containing in its relative interior only one point of the fixing system). 
Consider now the case md $M=3$ and denote by $M \subset R^{n}$ the body $M_{3} \oplus I \oplus \cdots \oplus I$, where $M_{3}$ is a three-dimensional body that is the convex hull of a ball and one of its external points, whereas each summand $I$ is a segment (and the number of the summands $I$ is equal to $n-3$ ). Then $M$ is a compact, convex body with md $M=3$ and $\operatorname{dim} M=n$. At the same time $\varrho_{\max }(M)=\infty$ (see [5] or [12]), i.e., the upper bound indicated in the main theorem is attained. A similar example may be constructed for any md $M \geq 3$.

To finish the proof, it remains to establish that the estimates, indicated in the main theorem for the case md $M=2$, indeed, are the exact upper bounds. Let $M \subset R^{n}$ be a compact, convex body with md $M=2$ and let $M=M_{1} \oplus \cdots \oplus M_{k}$ be its decomposition into the direct vector sum of indecomposable compact, convex sets. Then

$$
\varrho_{\max }(M)=\varrho_{\max }\left(M_{1}\right)+\cdots+\varrho_{\max }\left(M_{k}\right)
$$

(see the proof of Theorem 21 in [4]). Moreover, for each set $M_{i}, i=1, \ldots, k$, the following alternative opportunities are possible:

(a) $\operatorname{dim} M_{i}=1$, i.e., $M_{i}$ is a segment and hence $\varrho_{\max }\left(M_{i}\right)=2$.

(b) $\operatorname{dim} M_{i}=2$, i.e., $M_{i}$ is a two-dimensional convex set distinct from a parallelogram; in this case $\varrho_{\max }\left(M_{i}\right) \leq 6$ (see (1)).

(c) $\operatorname{dim} M_{i} \geq 3$, and $M_{i}$ is a stack, outcut, or stack-outcut. In this case $\varrho_{\max }\left(M_{i}\right) \leq$ $2 \operatorname{dim} M_{i}$ (see Lemma 2).

(d) $M_{i}$ is the special four-dimensional polytope (see Theorem 29.7 in [7]). In this case $\varrho_{\max }\left(M_{i}\right)=18$ (by Lemma 3).

If for an index $i$ case (b) is realized with $\varrho_{\max }\left(M_{i}\right) \leq 5$, then we may replace $M_{i}$ by a hexagon $H$ with pairwise parallel opposite sides, increasing the number (11). Thus to obtain the maximal value of (11), we have to suppose that every $M_{i}$ with $\operatorname{dim} M_{i}=2$ is a hexagon with pairwise parallel opposite sides, and hence $\varrho_{\max }\left(M_{i}\right)=6$.

By (11), we now have

$$
\varrho_{\max }(M)=2 q_{a}+6 q_{b}+2 D+18 q_{d},
$$

where $q_{a}, q_{b}, q_{d}$ are the numbers of the summands in (11) for which, respectively, cases (a), (b), (d) are realized and $D$ is the sum of dimensions of the summands $M_{i}$ for which case (c) holds.

Assume that for an index $i$ the summand $M_{i}$ is an $n_{i}$-dimensional stack, outcut, or stack-outcut $\left(n_{i} \geq 3\right)$. Then we may replace it by $M_{i}^{\prime}=H \oplus I \oplus \cdots \oplus I$ where $H$ is a hexagon with pairwise parallel opposite sides and the number of the segments $I$ is equal to $n_{i}-2$. By this replacement, the number (12) increases, since $\varrho_{\max }\left(M_{i}^{\prime}\right)=2 n_{i}+2>$ $\varrho_{\max }\left(M_{i}\right)$. This means that to obtain the upper bound it is necessary to suppose that there is no summand $M_{i}$ for which case (c) holds, i.e., $D=0$.

We see that to obtain the upper bound the body $M$ has to be the direct sum of the following form:

$$
M=I \oplus \cdots \oplus I \oplus H \oplus \cdots \oplus H \oplus \Pi \oplus \cdots \oplus \Pi,
$$

where $\Pi$ is the special four-dimensional polytope. Now we notice that if the number of the summands $H$ is greater than 1, then we may replace $H \oplus H$ by $\Pi$, increasing the 
number (12). Similarly, if the number of the summands $I$ is greater than 1 , then we may replace $I \oplus I$ by $H$, again increasing the number (12).

Consequently, for attaining the upper bound only the following four cases are possible:

$$
\begin{aligned}
& M=\Pi \oplus \cdots \oplus \Pi, \\
& M=I \oplus \Pi \oplus \cdots \oplus \Pi, \\
& M=H \oplus \Pi \oplus \cdots \oplus \Pi, \\
& M=H \oplus I \oplus \Pi \oplus \cdots \oplus \Pi .
\end{aligned}
$$

These cases correspond, respectively, to the possibilities

$$
\begin{array}{llll}
\operatorname{dim} M \equiv 0 & (\bmod 4), & \operatorname{dim} M \equiv 1 & (\bmod 4), \\
\operatorname{dim} M \equiv 2 & (\bmod 4), & \operatorname{dim} M \equiv 3 & (\bmod 4)
\end{array}
$$

and give the equalities (1)-(3). For example, in the case $n=\operatorname{dim} M=4 p+3$ (see (13)), we have

$$
\varrho_{\max }(M)=18 p+8=18\left(\frac{n-3}{4}\right)+8=\frac{9}{2} n-\frac{11}{2},
$$

accordingly the second formula (7). The other cases are considered analogously.

Remark. Combining the main theorem with inequality (3), we obtain the following corollary: Let $M \subset R^{n}$ be a compact, convex body with $\mathrm{md} M=2$. Then the cardinality of any primitive fixing system $F \subset$ bd $M$ satisfies the inequalities

$$
\frac{3 n}{2} \leq \operatorname{card} F \leq \frac{9}{2} n+\alpha_{n},
$$

$\alpha_{n}$ being defined by (6) and (7). We remark that not every integer satisfying these inequalities can be the cardinality of a primitive fixing system. For example, if $\operatorname{dim} M=4$ and $m=2$, then only the integers $3, \ldots, 13,15$, and 18 can be the cardinalities of primitive fixing systems, since from the proof of Lemma 3 it is possible to conclude that in the case $M=\Pi$ there is no primitive fixing system of cardinality 14,16 , or 17 . Indeed, if instead of a segment $I_{j}^{+}$or $I_{j}^{-}$we consider one of its endpoints (as the set $B_{j}^{+}$or $B_{j}^{-}$), then the cardinality of the corresponding primitive fixing system is no greater than 15 ; for example, if we replace the segment $I_{1}^{+}$by its endpoint $e_{1}$, then, by primitivity, the segments $I_{5}^{+}, I_{6}^{+}$, and $I_{9}^{+}$can be thrown out, i.e., we obtain the cardinality 15 (instead of 18).

\section{Acknowledgment}

The authors thank Professor Branko Grünbaum for kindness, attention, scientific conversations and, in connection with his birthday, wish him health, happiness, and success during many years. 


\section{References}

1. Bollobás, B. Fixing systems for convex bodies. Studia Sci. Math. Hungar. 2 (1967), 351-354.

2. Boltyanski, V.G. Helly's theorem for $H$-convex sets (in Russian). Dokl. Akad. Nauk SSSR 226(2) (1976), 249-252. (See also Soviet. Math. Dokl. 17(1) (1976), 78-81).

3. Boltyanski, V.G. A new step in the solution of the Szökefalvi-Nagy problem. Discrete Comput. Geom. 8 (1992), 27-49.

4. Boltyanski, V., and Martini, H. Combinatorial geometry of belt bodies. Results Math. 28 (1995), 224-249.

5. Boltyanski, V., and Martini, H. On maximal primitive fixing systems. Beiträge Algebra Geom. 37(1) (1996), 199-207.

6. Boltyanski, V., and Martini, H. Fixing and hindering systems in combinatorial geometry. Beiträge Algebra Geom. 40(2) (1999), 551-563.

7. Boltyanski, V., Martini, H., and Soltan, P. Excursions into Combinatorial Geometry. Springer-Verlag, New York, 1997. xiii +419 pp.

8. Boltyanski, V.G., and Morales Amaya, E. Minimal fixing systems for convex bodies. J. Appl. Anal. 1(1) (1995), 1-11.

9. Danzer, L. Math. Reviews 2942 (1963), 569-570.

10. Fejes Tóth, L. On primitive polyhedra. Acta Math. Acad. Sci. Hungar. 13 (1952), 379-382.

11. Grünbaum, B. Fixing systems and inner illumination. Acta Math. Acad. Sci. Hungar. 15 (1964), 161-168.

12. Morales Amaya, E. A note on maximal primitive fixing systsms for convex bodies. Note Mat., to appear.

13. Szökefalvi-Nagy, B. Ein Satz über Parallelverschiebungen konvexer Körper. Acta Sci. Math. 15(3-4) (1954), 169-177.

Received October 14, 1998, and in revised form May 5, 1999. Online publication May 3, 2000. 